\title{
Bildgebung (INMB)
}

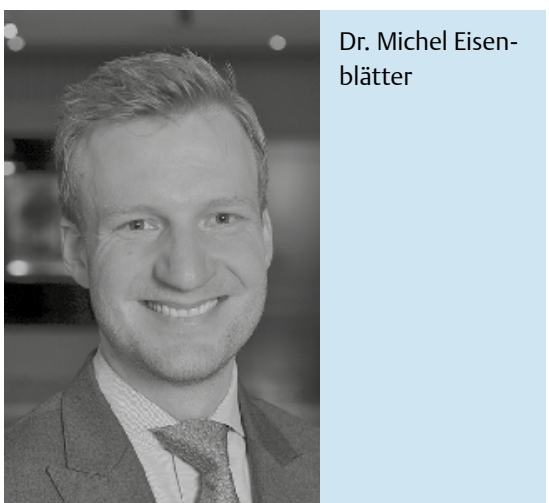

Sehr geehrte Damen und Herren, liebe Kolleginnen und Kollegen,

derzeit beobachten wir eine rasante Entwicklungen im Bereich neuartiger Therapien von Krebs und der vaskulären und neurodegenerativen Erkrankungen, oft getrieben durch Erkenntnisse aus der Genomik, Proteomik oder Immunologie. Klinisch verfügbare diagnostische Verfahren, die es erlauben würden, solche Therapien zu verfolgen oder die Patienten dafür zu selektieren hingegen fehlen häufig noch bzw. sind unzureichend.

In der Grundlagenforschung erlebt die Bildgebung - für Diagnostik ebenso wie

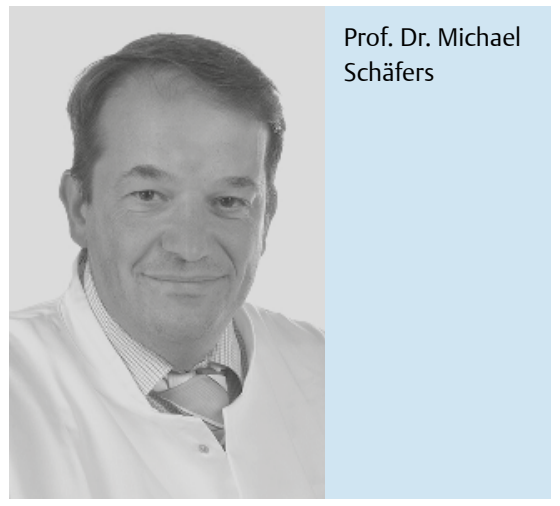

zur Entschlüsselung pathophysiologischer Prozesse - eine ständige Weiterentwicklung und Anwendung.

Die Bildgebungsforschung bietet damit die große Chance, Techniken und Erkenntnisse aus der präklinischen Grundlagenforschung in die klinische Anwendung zu übertragen. Die mit einer Translation verbundenen Herausforderungen und Chancen für das Feld der Molekularen Bildgebung stehen im Mittelpunkt der MoBi16. Wir freuen uns, Sie zur MoBi16 im Oktober nach Münster einladen zu können. Die MoBi bietet ein in dieser Form einzigartiges Forum. Wissenschaftler aus der

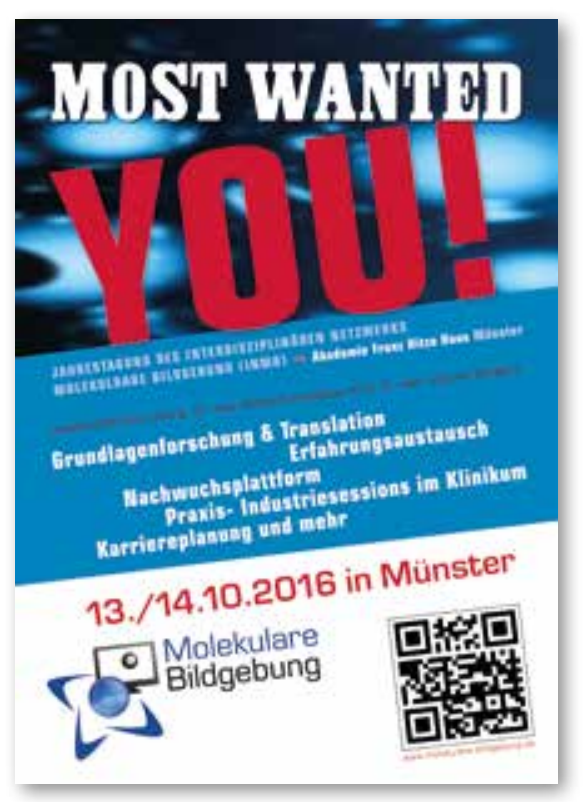

Grundlagenforschung, klinische Anwender und Entwickler aus der Industrie treffen sich hier zum Erfahrungsaustausch und zur Diskussion auf nationaler Ebene. Die MoBi hat sich insbesondere für den wissenschaftlichen Nachwuchs zu einer wichtigen Plattform dafür entwickelt, die eigene Arbeit zu präsentieren und ein eigenes Netzwerk aufzubauen.

An diese Tradition möchten wir auch in Münster anknüpfen. In spezialisierten Sessions werden wissenschaftliche Beiträge durch Übersichtsvorträge etablierter Wissenschaftler eingeführt. Hierbei werden Experten aus der Grundlagenforschung, der Methodenentwicklung und der klinischen Medizin zu Themen der Onkologie, Immunologie, kardiovaskulären Medizin, Technologieentwicklung und Neurowissenschaften zu Wort kommen.

Zur Diskussion mit den Fachleuten aus Wissenschaft und Industrie wird zudem außerhalb der Vortragssessions beim „Meet the Expert“ Scientific Speed Dating und bei der gemeinsamen Abendveranstaltung Gelegenheit sein.

Zum 1. Mal wollen wir auch die Möglichkeit schaffen, die Innovationen aus der Geräteentwicklung der Industrie in dezidierten Sessions live im Einsatz zu demonstrieren.

Wir freuen uns darauf, wenn Sie unserer Einladung nach Münster folgen und im Oktober Teil der MoBi16 werden!

\section{Herzlichen Gruß}

Dr. Michel Eisenblätter,

Prof. Dr. Michael Schäfers 